despite its enormous past contributions to our economies. This was reflected in the applications received: industrial participation was minimal. Excuses can be made for this but surely we need to build, or rebuild, bridges over the gulf between us and our industrial colleagues. What better way than with a network, under whose auspices the two sides can identify and develop their shared interests?

Stimulation, SCIENCE and HCM (TMR's predecessors) and TMR itself have made a unique contribution to demolishing barriers between scientists. National funding agencies remain trapped in chauvinist mentalities, for the most part. TMR lets a lucky few spread their wings. Its continuation into the next Framework Programme deserves strong political support.

\title{
Training and Mobility of Researchers
}

\section{Networks to Train}

The European Union (EU) Training and Mobility of Researchers (TMR) Programme, part of the EU's 4 th Framework Programme 1994-98, comprises four activities (networks, training grants, access to facilities, and conferences). Networks in its first round that closed in June 1995 netted 1427 eligible proposals. Following review by the seven disciplinary panels, only $91(6.4 \%)$ were selected, largely owing to budgets designed to increase significantly the average funding per network from the 250-500 kECU range typical for networks in TMR's predecessor (Human Capital and Mobility - HCM) so as to provide more depth, such as support for postdocs.

The TMR first round involved a larger number of proposals than the equivalent stage in HCM (HCM received some 2199 in its entire operational period of about 3 years) because fields such as economics are now eligible and others have gained experience, because teams make several proposals, and because the delayed launching of TMR led to a builtup demand.

A total of 332 proposals (close to the equivalent HCM level of some $25 \%$ ) were in physics, and only $6 \%$ were selected (as compared to over $30 \%$ for $\mathrm{HCM}$ ). Peter Kind, who manages the network activity, has indicated that networks were not selected on the basis of targeted fields since targeting has not been chosen as an eligibility criterion by the Council of Ministers. It is difficult to define and set targets in science in a programme that is intended to be bottom-up (i.e., based on projects put forward by scientists).

TMR has decided to combine the final two selection rounds, to reduce the period for a network from 4 to 3 years (justifiable since 3 years still gives continuity into the 5 th Framework), and to reduce the funding per team per year from 50-80 kECU to $40-70 \mathrm{kECU}$ (mostly by reducing salary support for experienced scientists so as not to diminish the emphasis on the training of young people). The result will be a further 160 networks funded, with an average of 1.25 MECU per network (as compared to $1.6 \mathrm{MECU}$ for the first round) and about 25 postdocyears per network (instead of the present 30).

Some $60 \%$ of a typical network's salary costs are for training young scientists. So applicants will be asked to explain why training is needed. Networks are usually multidisciplinary. But it will not be necessary for each individual component of a network to justify why its particular area needs trained people. It will instead be the overall picture that is considered. Peter Kind indicates that in addition to offering real interaction at the working level, networks should provide training opportunities for young scientists (not simply employment for a pair of young hands). The training should benefit from being inside a multidisciplinary, international project involving complementary teams so that young collaborators encounter a broad range of challenging problems.

The guidelines for TMR networks in the next (and last) selection round closing on 16 September 1996 are: Size - at least 5 research teams, 3 countries, $1 \mathrm{EU}$ team, and 3 established teams. Financing $-100 \%$ additional costs; 40-70 kECU/team/year; up to $15 \mathrm{MECU}$ total; up to 3 years; no more than $40 \%$ of finance to teams in one country.
Grants Underexploited

Framework programmes other than TMR offer mobility grants ( 118 were awarded in early 1996 , on the same terms as TMR Research Training Grants, by six specific programmes). The EC advises applicants to contact programme officers to see if it best to apply to a specific programme or to TMR.

TMR of course offers by far the largest number of fellowships. Of the roughly 5700 eligible proposals dealt with in the first three rounds (June, September December 1995 calls), some 1100 were in physics; they had a success rate of $22 \%$ for the first two rounds.

Barry McSweeney, who was recently appointed Head of the TMR Fellowship activity, says that reducing the time to process an application to 5 months has decreased significantly the number of complaints (a limit of 4.5 months is determined by the EC's procedures). Awards are sent out by institutes, and the first are now reaching successful applicants.

For the first two rounds, postdocs accounted for $54 \%$ of eligible applications ( $23 \%$ in physics) of which $25 \%$ ( $26 \%$ for physics) were successful; predocs accounted for $42 \%$ eligible applications ( $19 \%$ in physics) of which only $11 \%$ (10 \% for physics) were successful. So postdocs are more successful than predocs, reflecting Council's policy that TMR grants be aimed at postdocs, and the inclusion of economics has tended to increase the number of predoc applications.

What is surprising is the poor response by experienced scientists. Only 95 applied in the first two rounds and a massive $82 \%$ were successful. Many more could have been funded.

The impact of TMR grants is judged to be considerable and fellows are being honoured by naming them Marie Curie Fellows. A major concern has been that TMR grants stimulate a brian drain to prosperous regions, so one-year return grants to help resettle postdocs have been created. In the first two rounds, 20 eligible applications were in physics and $85 \%$ were successful.

The next TMR training grant call closes on 15 June, with the following calls as planned every six months. Some Institutional Fellowships left over from $\mathrm{HCM}$ can be applied for until 7 June. Applicants must first approach a preselected institute. The EC says it will publish the list of institutes on WWW, presumably via the TMR homepage (http://www2.cordis.lu/ $\mathrm{tmr} / \mathrm{src} / \mathrm{tmr})$.

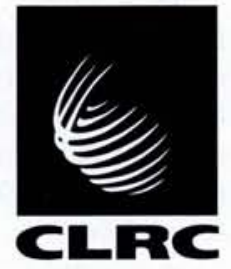

CALL FOR PROPOSALS

\section{EU - Funded Access to the Central Laser Facility Rutherford Appleton Laboratory}

The Central Laser Facility at the Rutherford Appleton Laboratory operates one of Europe's premier laser laboratories for research in pure and applied science including ICF, $x$-ray lasers, basic plasma physics, chemistry, biology and x-ray source applications.

Facilities include large scale $\mathrm{Nd}$ :glass and $\mathrm{KrF}$ lasers delivering focused intensities in excess of $10^{19} \mathrm{~W} . \mathrm{cm}^{-2}$, a dual-wavelength tuneable OPO system for ultra fast spectroscopy, a 110ps resolution confocal microscope and a $10 \mathrm{~Hz}$ laser plasma $\mathrm{x}$-ray source. See also http://www.nd.rl.ac.uk/lasers/

Applications for beam time are invited from EU and UK based scientists for experiments in the period July December 1996.

Contact Chris Edwards for further details of the facilities available and an application form.

E-mail: c.b.edwards@rl.ac.uk; $\quad$ Fax: +44 (0) 1235445888

Complete applications must be received by 22 April 1996 . 